\title{
Using Tweets to Support Disaster Planning, Warning and Response
}

\author{
Peter M. Landwehr, Wei Wei, Michael Kowalchuck \\ and Kathleen M. Carley ${ }^{1}$ \\ \{plandweh, weiwei, kf3cr, kathleen.carley\}@cs.cmu.edu \\ Carnegie Mellon University \\ School of Computer Science \\ Institute for Software Research \\ 5000 Forbes Avenue, \\ Pittsburgh, PA 15213
}

${ }^{1}$ Corresponding Author: Prof. Kathleen Carley (kathleen.carley@cs.cmu.edu), Institute for Software Research, Carnegie Mellon University, Pittsburgh, PA 15213 USA. 


\section{Using Tweets to Support Disaster Planning, Warning and Response}

\section{1 - Introduction}

Social media in general, and Twitter in particular, provides a rapid approach to gathering crowd-sourced information and a way of reaching many members of the population. As such, it can be harnessed to support warning and response efforts for extreme events, such as Tsunamis. In this paper, we present a Tsunami Warning and Response Social Media System, TWRsms, a Twitter based web-application component for a socio-technical decision support system for extreme events - see http://legion.casos.cs.cmu.edu:8011/. TWRsms is tailored to a specific community, Padang Indonesia, and a specific type of event - Tsunamis. Indonesia is an ideal location for testing and developing a Twitter-based alert system for tsunamis for two reasons: vulnerability to disaster and widespread adoption of the social media platform.

This paper is part of a special issue on building community resilience to hazards. TWRsms is designed to work interoperably with the DDSS system described by Ai et al. (this issue), provide baseline information for the opportunistic routing prior to power outages (Santos et al., this issue), and will switch modes when alerted by a detection system which will be informed by the detection model (Xerandy et al. this issue). Further it will provide up-to-date twitter population information to a community system. The overall system, of which TWRsms is a component, is designed to be used in addition to existing technology to provide more timely warnings and to reach a larger fraction of the community at risk. In addition, since TWRsms stores historical information and supports tweet categorization it can be used to support planning and response efforts. Although, herein, we will not focus on the use of TWRsms for planning and response. TWRsms, though tailored to Padang, was built to be general purpose. Hence, while the reader may find the features of this system of interest, system designers may find the general guidelines for developing a social media system for tsunami warning and response even more valuable. TWRsms is available at the CASOS website http://legion.casos.cs.cmu.edu:8011/.

In order to develop this system and to tailor it to Padang we first needed to understand the affordances of Twitter in general for the various phases of disasters, and the way in which the Padang community uses Twitter. These factors impact how Twitter data should be collected so as to best support the early warning efforts. This information is described by Carley et al, (this issue).

How can social media be harnessed to mitigate the impact of natural disasters? Social media is useful for disasters because it supports community resilience and engagement by allowing information gathering to be crowd-sourced thus supporting broader, less costly and more current data collection either from those affected or those collecting data. Social media provides responders with an additional media for getting information out to the public, and that information is sent in a way that it is easy to "forward" to others thus increasing the rate of dissemination. Social media can capture relevant information for mitigating disasters such as images, locations, and numbers of people.

Currently, there are a variety of efforts to employ social media at different phases of a disaster (Coppola, 2015). On the one hand, various agencies are creating a presence in social media so that they can provide warnings and updates to the public through these 
media (Basher, 2006). For example, in Indonesia, BMKG (@infoBMKG) provides early warning on Tsunamis via Twitter (Chatfield \& Brajawidagda, 2012). On the other hand, there is a growing interest in using social media for disaster response (Gao et al, 2011). For example, after Hurricane Sandy, Occupy Sandy - a loosely organized group of former Occupy Wall Street activists and other volunteers, used social media and a website to coordinate efforts and bring food and supplies to those in need. However, social media has not been effectively employed as part of the early warning phase or the planning phase. TWRsms enables Twitter to be harnessed during these two phases. During early warning and planning, the way in which Twitter is normally used by the population is more critical than the peculiarities of Twitter response during or in the immediate aftermath of a disaster. Hence, an analysis of normal Twitter usage for the region, see Carley et al this issue, is critical.

In general, all Twitter applications for disaster, regardless of the phase of disaster they seek to support, entail collecting and rapidly analyzing large numbers of tweets. Most systems employ a basic workflow of collection, analysis and presentation. This means that the applications tend to be one-off and have little reuse and that the data is not available for planning or research. In contrast, we suggest that to truly support disaster mitigation across all phases, a better workflow is:

1. Collection, in which tweets are collected.

2. Management, in which tweets are archived and reduced in size.

3. Analysis, in which different analyses are run on the reduced tweets.

4. Presentation, in which the analyses are presented for assessment.

5. Integration, in which the Twitter workflow is linked into a larger disaster system.

TWRsms uses this workflow. In this paper, we describe TWRsms and use it to illustrate for system developers what is entailed at each step in the proposed workflow, what the issues are and what features are needed. To orient the reader, we begin by describing why Twitter will be a useful component of a disaster mitigation plan in Indonesia and how the current Indonesia disaster mitigation efforts use Twitter. We then describe our system and provide additional details on the workflow from both a general (any system) and a specific (our system) perspective. We follow this section with a description of the data collected by the system (tweets) and its relative value for mitigating the effects of disasters. We conclude by describing ongoing challenges posed in leveraging Twitter data for disaster support.

\section{2 - Indonesia - Disasters \& Twitter}

In Indonesia, frequent natural disasters coupled with the extremely weak infrastructure has created a situation in which any support to mitigate disasters can have major consequences. The specific infrastructure weaknesses, such as roads and public broadcasting, means that it is difficult to disseminate information which can lead during a disaster to loss of life. In general, reactions to disasters are generally ad hoc and disorganized (Earthquake Engineering Research Institute, 2009). Anecdotal evidence from the last two major disasters in Indonesia make it clear that that the entire population at risk were not being reached. For example, early Tsunami warnings did not reach large segments of the population resulting in deaths. New means of communicating with the public may help to fill this gap. 
In Indonesia, great efforts are underway to make use of the internet and social media to mitigate disasters. For example, relatively up-to-date information on Indonesian disasters can be found on the official website of the Indonesian National Disasters Mitigation Agency - the Badan Nasional Penanggulangan Bencana. ${ }^{1}$ When a disaster hits the lead agency is the National Disaster Management Agency (Badan Nasional Penanggulangan Bencana or BNPB). This agency works closely with the Meteorological, Climatological and Geophysical Agency of Indonesia (Badan Meteorologi, Klimatologi, dan Geofisika or BMKG). BMKG is the lead Indonesian agency for tsunami detection and early warning for these natural disasters. It operates the Indonesia Tsunami Early Warning System (Ina TEWS) which is used to detect, analyze, simulate, and disseminate disaster information on earthquakes, tsunamis, and other severe weather conditions. Chatfield and Brajawidagda (2013) in a detailed assessment of this system find that a Twitter based warning system could be a viable complement to the existing Ina TEWS system.

Increasingly Indonesians are spending money on cellphones and particularly low-cost smartphones. ${ }^{2}$ Indonesians are spending time on social media - both from their cell phones and from other internet access ports. Indonesians have proven to be prolific adopters of Twitter, with an estimated 29.4 million users in 2012 (Semiocast, 2012). Based on World Bank estimates that $15.4 \%$ of Indonesia's population has Internet access and a 2012 population of 247 million, 77.29\% of Internet users are using Twitter. For additional details see Carley et al, this issue. Consistent with earlier instances of Indonesians improvising with community radio to disseminate information in disaster situations, there is anecdotal evidence that they also use Twitter in disaster situations. The interest in Twitter usage for disaster response is so strong in Indonesia that there are currently several proposals to develop a tsunami early warning system for Indonesia using Twitter (Birowo, 2010; Anggunia \& Kumaralalaita, 2014; Chatfield \& Brajawidagda, 2012).

Government agencies in Indonesia are avid Twitter users. The disaster related agencies are no exception - see for example@BMKGIndonesia,@BNPB, and@NewEarthquake. These agencies have a high number of followers and are frequently retweeted. For example, in $2012 \mathrm{BMKG}$ had over 300,000 followers and now has over 1 million. In 2012, BMKG was retweeted on average 2000 times per message. For tsunamis BMKG sends out its first alert 5-7 minutes after the detection of the tsunami causing earthquake.

\section{3 - The Padang Twitter Tsunami System}

To develop a Twitter Tsunami System for Padang we compared and contrasted a number of existing tools, tested multiple approaches to data collection, identified core needs from the literature and discussions with responders, and discussed local needs with members of the Indonesian disaster response community. This study resulted in a set of goals for the TWRsms:

- Scalable to multiple types of social media

- Scalable in terms of data volume

- Rapid processing for material useful in early warning

- Support for planning

\footnotetext{
1 http://www.bnpb.go.id/ Accessed February 2015

${ }^{2}$ http://www.voanews.com/content/in-indonesia-a-smartphone-for-every-budget/1628734.html Accessed February 2015
} 
- Support for early warning

- Support for response and recovery

- Interoperable with existing and proposed systems

- Generalizable to areas other than Padang

- Data survivability in a disaster

- Ease of access

To meet these goals TWRsms is a general-purpose, scalable and extensible web-based system accessible from smartphones, tablets or computers to support ease of use. The front page for the working system is shown in Figure 1. We have used this system to: 1) identify procedures for processing and presenting data that are fast, scalable and easy to use; 2) demonstrate to people in Padang how Twitter can be used as part of a Tsunami Warning and Response system; and 3) capture twitter data to support planning. The system is built with interoperability in mind so that it can be use in concert with the other technologies described in this issue. New social media, or multiple Twitter draws can be added to support broader data collection. To apply TWRsms to an area other than Padang required only redirecting the Twitter data pulls and using shape files for the region of interest. Before such a system would be used insitu an official organization responsible for maintenance and pressing the alert signal would need to be identified and the system transferred to them. We are working with the local Indonesian group headed by Harkunti Rahayu to find such an organization. Such an organization would need to have sufficient computer storage, access to remote site secondary backup for data survivability, authority to send alerts, and so one. Herein, we describe only the technical issues, and not organizational issues in using and developing TWRsms.

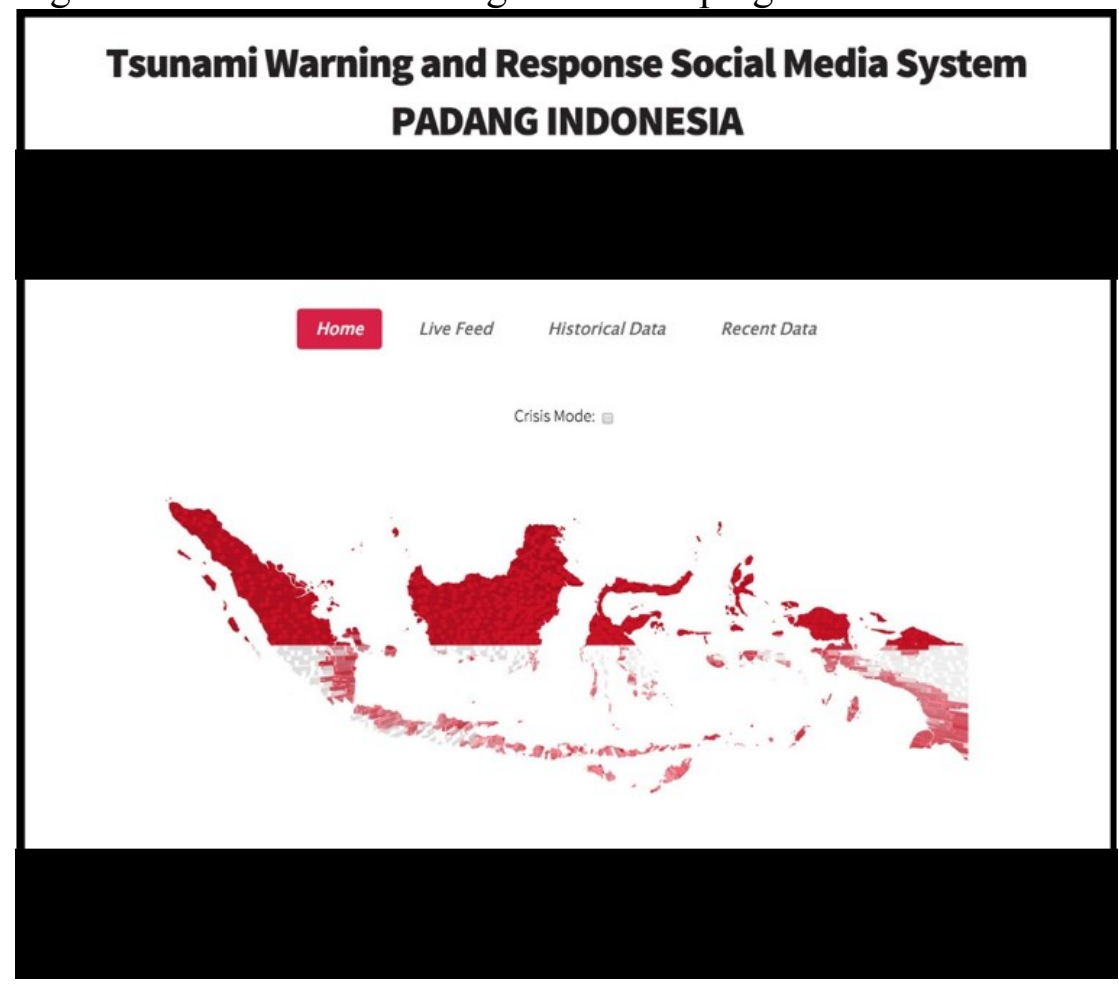

Figure 1. Webpage front end for the Padang Twitter Tsunami System 
TWRsms is designed to operate in two modes, disaster alert and normal. In the disaster alert mode based on an external signal, the operator hits the disaster alert button and a tweet is sent out to the public and to other systems in the overall water-land system and specifically to the Raspberry Pie system. In addition the viewable web page then changes to the current tweets and a projection for the next hour based on historic data for this day of week and hour. In the normal mode there are three sub-pages: Live Feed - which shows Twitter traffic by official sources related to Tsunamis; Historical Data - which presents the analysis of data collected for the region over the past years; and Current Data - which presents the analysis of the data collected in the last seven days.

Live view is one of the ways in which TWWRsms is interoperable with existing systems and supports early warning. This pulls from existing official sources, and creates a single stop mashup of the results. In Live view the user can link to the official sources and follow them directly. In Figure 2, the Live view page for a random day is shown. Note the content will change continuously as only the most current tweets are shown.

Historical and current data are both reporting on the collected twitter data. The historical data has a combination of a 10\% data pull from 2011-2013, merged with the ongoing $1 \%$ feed. The system is designed so that if the official group would maintain the site has able to same fashion. Further, to support ease of use, both the historical and current pages have many of the same representation schemes for data e.g., bar charts and heat maps. For both historical and current many of the same network metrics are calculated and stored. Both also export data in CSV format to support interoperability. The key difference is that in the historical system, the full tweet and all details on the tweeter are not stored, only summary data. Hence, if a Tweeter changes their privacy settings, we do not need to re-clean the data, as no personally identifiable information is stored.

The historical data section of TWRsms supports planning, and potentially research on social media use in disasters. In Figure 3, an example map and bar chart from the Historical data webpage is shown. The user can change what region for which data is shown by selecting to the region of interest (e.g., by selecting a name or clicking on an area). The user can also choose to see data distributions by day of week (collapses across hours), hour of day (collapses across days), or hour of day for a week.

The current data section supports early warning, immediate response and recovery. Under the current data section the user can see where people are tweeting from, the influential Tweeters overall or by region, and the common hashtags by region or overall. It will eventually support identification of those tweets focused on disaster related issues. The system is designed to report data as counts, percentage of Tweeters/Tweets, in comparison to historic data, and as percentage of the population thought to occupy that region. One of the most interesting ways in which data is presented for ease of use is as a heat map. In Figure 4, an illustrative heat map for Padang is shown, showing where the Tweets from those Tweeters who have their geo-codes enabled are coming from. For officials this provides guidance for which areas can be reached by Tweets. It is important to note here that the coastal area is relatively low in Twitter activity; whereas, the highest activity is coming from the university regions. 


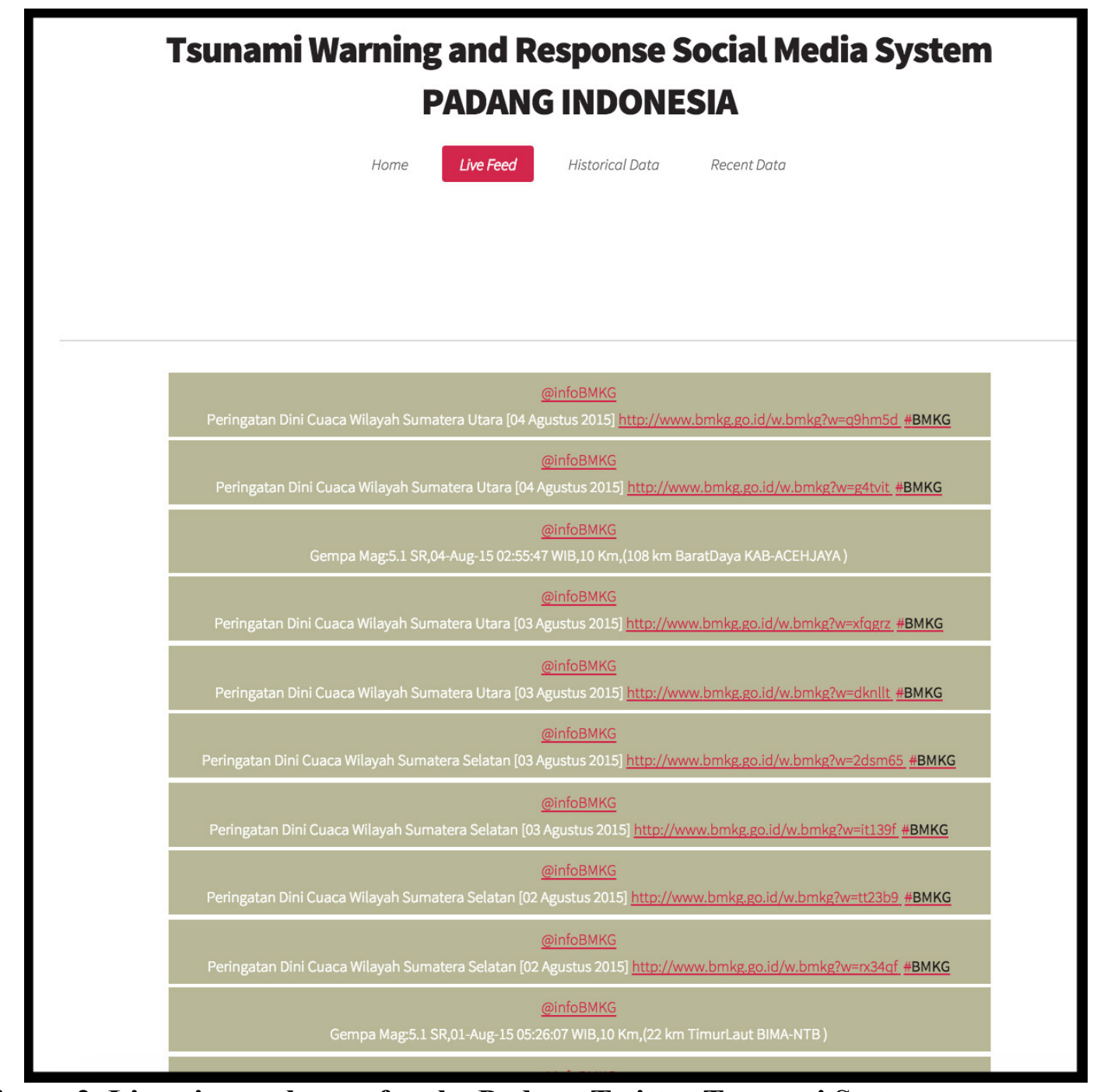

Figure 2. Live view webpage for the Padang Twitter Tsunami System 


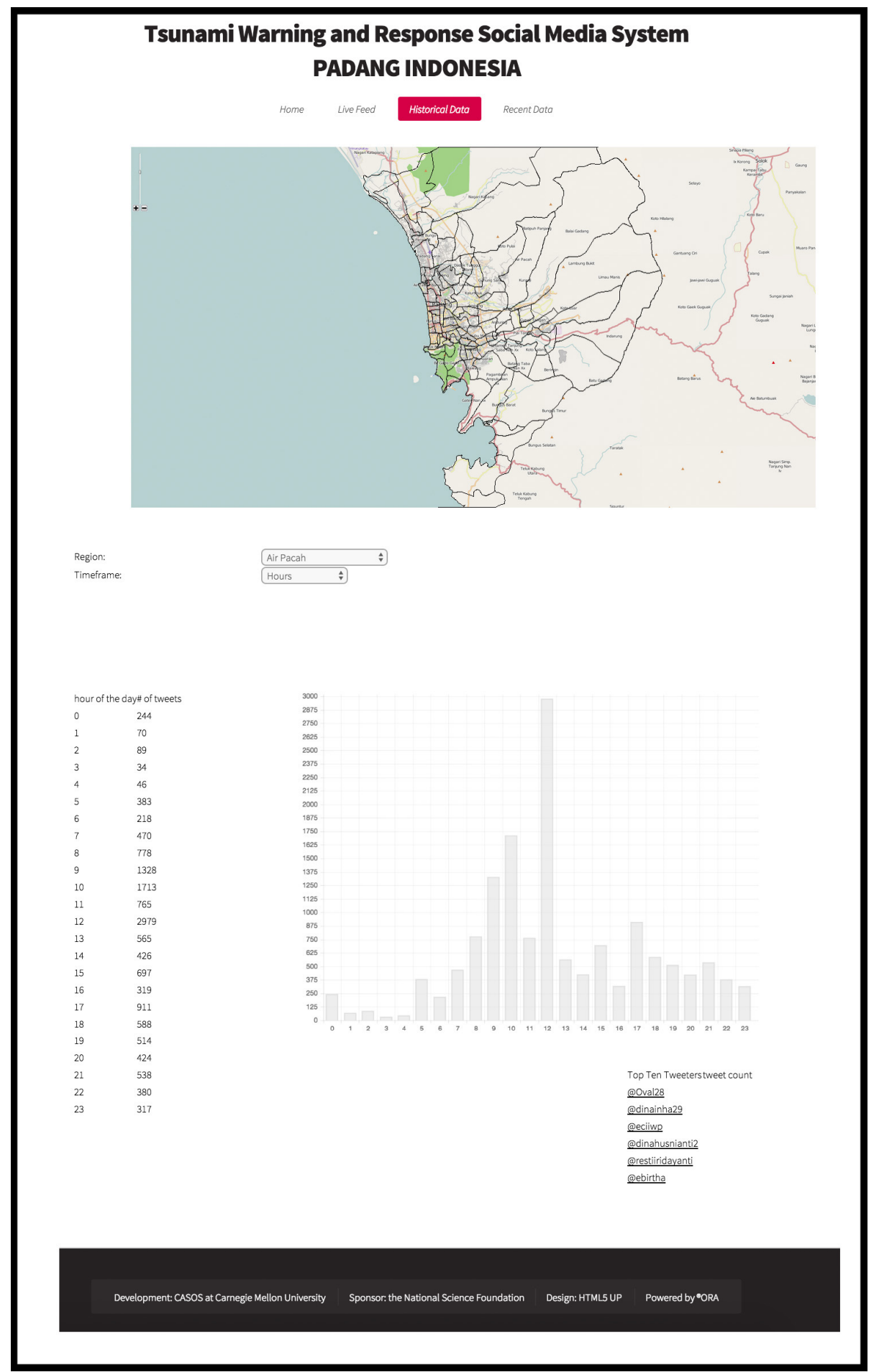

Figure 3. Illustrative webpage showing the presentation of historic data in bar charts in the Padang Twitter Tsunami System 


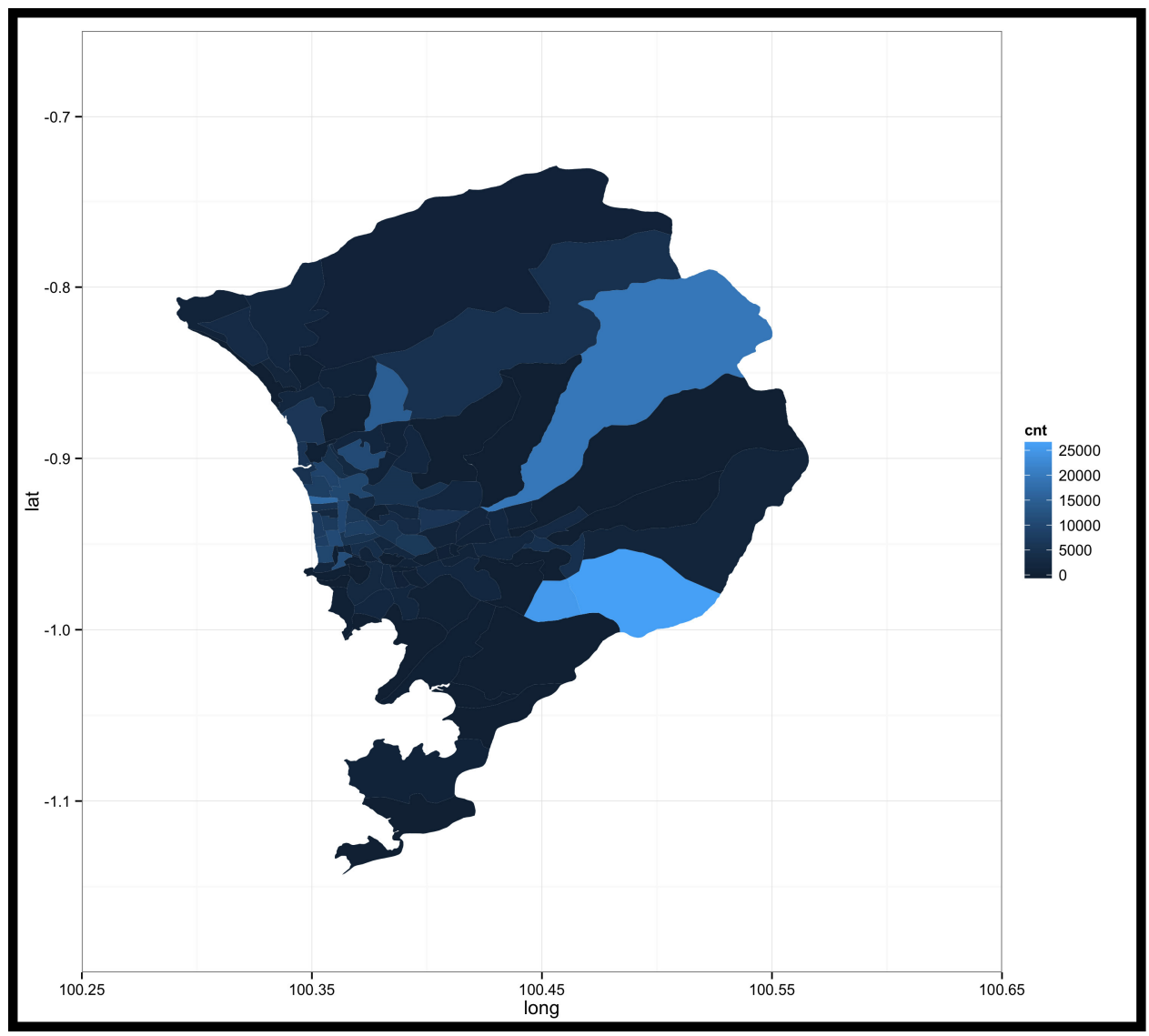

Figure 4. Image from the TWRsms webpage showing the current data as a heat map for Padang (lighter more tweets)

There are several key challenges in storing and retrieving data for this type of system. The foremost of these is that to use data from Twitter for research is allowed, but to share the tweets is not. Hence, data needs to be stored and shared in such a way that the original underlying tweet is not shared. A related constraint is that if a user of twitter removes personal information, then that information, even if stored separately cannot be shared which is a problem for historic data but not current data. These types of usage policy constraints exist for most social media. They limit the usage, storage, and sharing of the data. There are several implications. First, any historical data to be shared needs to be limited to summary information across the population, rather than features of individuals. Second, interoperation with other tools has to be done in terms of the summary data; i.e., csv files with means and averages, images of maps, and images of social networks.

\section{4 - Leveraging the Workflow}

TWRsms was developed as a confederation of sub-tools that support tweet collection, de-duplication, sorting, analysis and visualization. The analysis and visualization are done using the ORA tool (Carley et al, 2013; Carley 2014). Looking toward the future, increasingly systems for disaster mitigation, regardless of the phase, are systems of systems - involving loose confederations of interoperable component systems each of which can be used alone or in conjunction with others. This is particularly true for system used internationally. 
In this section, we describe both a general workflow for collecting, analyzing, and presenting tweets and our specific instantiation of this workflow, as well as the design issues and technologies used. TWRsms is an example of the proposed general workflow shown in Figure 5. This workflow has five components:

1. Collection, in which tweets are collected

2. Management, in which tweets are archived and reduced in size

3. Analysis, in which different analyses are run on the reduced tweets

4. Presentation, in which the analyses are presented for assessment

5. Integration, in which the system is treated as part of a larger disaster system

At the end of this process, raw Twitter JSON should have been extracted from the Twitter stream, reduced in size, archived, and changed into useful results.

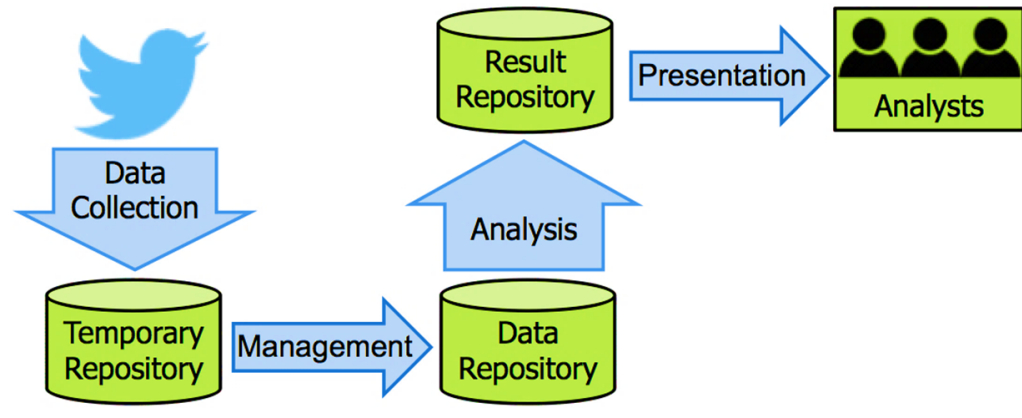

\section{Figure 5. Our \\ Generalized workflow for collecting, analyzing, and presenting tweets}

As we review each section of this workflow,

we provide specific keys into our implementations of each section. For Collection, we discuss our use of TweetTracker to aggregate tweets. For Management, we discuss our use of a collection of bespoke middleware for deleting unnecessary tweets from the data. For Analysis, we discuss our use of the ORA network analysis tool on our tweets. Lastly, for Presentation we discuss the website where we have stored our data. (See Figure 6.)

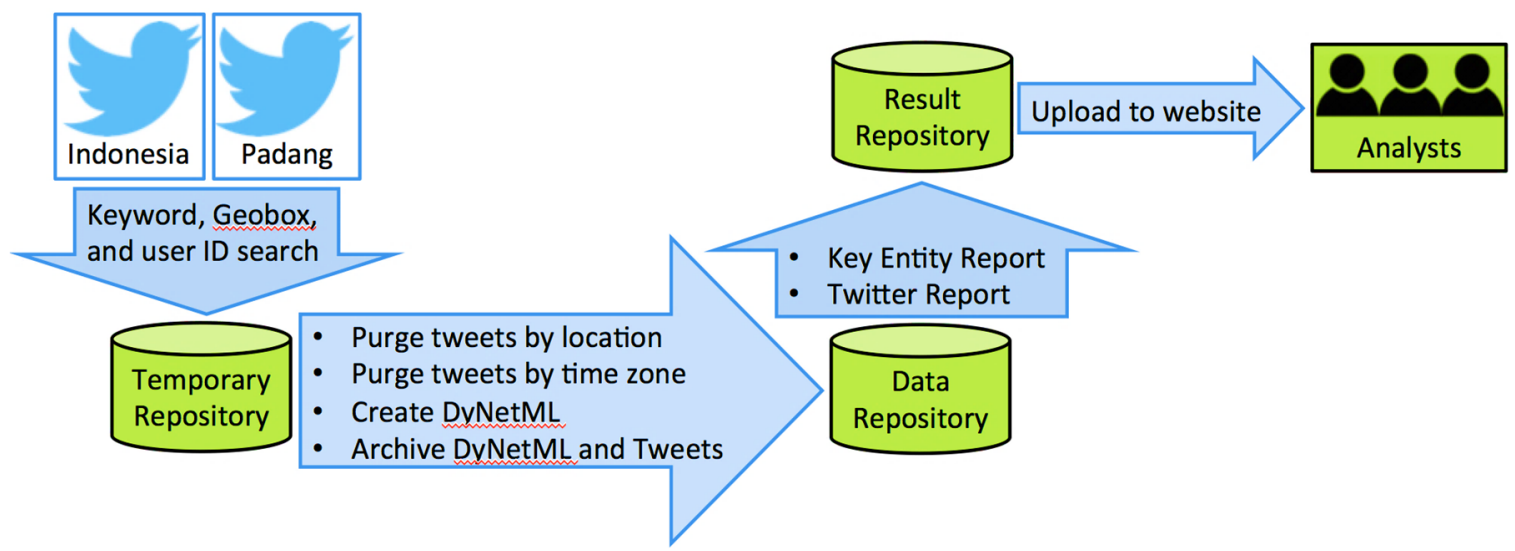

Figure 6. Our specific implementation of the general workflow, built using a combination of Python scripts, Java code, and the ORA network analysis tool.

\section{1 - Collection}

The fundamental component of any pipeline of tweets is a method of collecting tweets from Twitter's streaming API. Twitter provides documentation to help interested programmers develop tools for this interface (Twitter, 2012). They also provide a Java library that can be used as the basis for building queries (Twitter, 2014). Further, a number 
of free libraries have been built by developers to support accessing the API using other programming languages, including $\mathrm{C}++$, Python, and Ruby (see commentary by swatkat, et al.; aalronham; E.Michaels etc.).

If it is not possible to create a program for aggregating tweets from the Streaming API, a number of programs already exist to do so. Few of these are free; those that aren't often provide streamlined interfaces for analyses or provide support for connecting to other sophisticated analytic tools such as DataSift ${ }^{3}$, Topsy ${ }^{4}$, Texifter ${ }^{5}$, Simply Measured ${ }^{6}$, and HootSuite $^{7}$ (see O’Brien, 2013; Bruns \& Liang, 2012 for more information). If the implementation does not give raw Twitter JSON (JavaScript Object Notation) data, however, it will take you outside of the workflow described here.

In our implementation, we initially used TweetTracker, a tool developed by Arizona State University for collecting tweets from the streaming API (Kumar et al., 2011; Kumar $\&$ Morstatter, 2011). We use a command-line version of the tool to better integrate it with our workflow, but a version with a graphical user interface (GUI) also exists. When setting up this portion of the system, it is important to consider how interactive it is intended to be; the less interactive, the more it will be necessary to avoid GUIs and stick with fully automated processes. We recently switched to direct pulls from the Twitter API as this speeds up data collection and allows greater control over how the data is stored.

To determine how best to collect Twitter data for a region of interest for disaster planning and early warning a separate study was done focusing on the way in which Twitter is used in Indonesia (Carley et al, this issue). As part of that study a number of Twitter collection strategies were considered for Indonesia as a whole and for Padang in particular. This study showed that for a local region, such as Padang the best strategy is to use a npunding-box to first collect the tweets and then to remove those tweets that were not of interest using key words. The second best strategy is to collect with both keywords and a npunding-box. In Appendix A the keywords and npunding-box for Padang are provided. It is important to keep in mind that the exact data collection strategy will depend on whether the collector is buying the entire twitter firehose, the $10 \%$ decahose or using the $1 \%$ streaming API. In general, this latter choice is the most economical and is sufficient to meet disaster management needs (see Carley et a., this issue).

The streaming API provides a continuous stream of up to $1 \%$ of the total collection of all tweets being broadcast at a time, based on a set of search parameters provided by the user. The search API, which can be accessed from the Twitter website using a REST interface, allows searching on multiple parameters at once. This function poses its own set of challenges, and is challenging to use to scrape a large number of tweets over a sustained period. These parameters can include keywords, location npunding-boxes, and user IDs. (See Appendix A for the Padang parameters.) Tweets are collected if they contain any of the specified variables. In the Twitter API, if you specify both a geographic npunding-box and a list of keywords it is treated as an OR by Twitter and Twitter will provide tweets that are from either the region of interest, or that contain one or more of the specified keywords,

\footnotetext{
3 http://datasift.com/ last accessed February 2015

${ }^{4}$ http://topsy.com/ last accessed February 2015

${ }^{5}$ http://texifter.com/ last accessed February 2015

${ }^{6}$ http://simplymeasured.com/ last accessed February 2015

${ }^{7}$ https://hootsuite.com/ last accessed February 2015
} 
or some combination of location and words. Twitter does not support tweets to be selected based on an "AND" of the parameters.

The specific parameters we used for Padang are in Appendix A. This included a npunding-box that surrounded Padang. It is important to recognize that even when only a npunding-box is specified the data collection will include tweets from outside the political boundaries of the region of interest. There are two reasons for this. First, the npundingbox can only be specified as a rectangle and most political regions are not pure rectangles. Secondly, Twitter uses this npunding-box to identify which polygons to draw tweets from for the sample. Any Twitter defined polygon that overlaps the npunding-box will be used to select tweets from. Thus, multiple small npunding-boxes will help to ensure that the tweets are coming from all and only the region of interest.

As noted, we also examined the use of search terms both to collect tweets from the API or to remove unwanted tweets after they were collected. We use terms referring to specific geographic locations in Indonesia, Indonesian Slang terms for disaster and political issues, political terms and disaster related terms. For all terms we used both the English and Indonesian term as Carley et al, (this issue) showed that a significant fraction of tweeters in Indonesia and Padang tweeted in English. The disaster related terms were based on prior studies of how people referred to events in disasters, and were oriented around the Maslowian hierarchy of needs with a focus on food, water, shelter, missing individuals, and corruption. For Indonesia as a whole we also used the user ids of prominent political actors who would be expected to send official tweets when disasters occurred.

Padang is located on the west coast of Sumatra and is particularly vulnerable to tsunamis. By collecting tweets from both Padang and the Indonesian mainland, we hope to derive a set of distinctive features that contrast local and national reactions to tsunamis. TWRsms was designed to support such general comparison. Over time, we expect this will help us understand how to expand the system to a national system.

Data collection from the streaming API for Padang and Indonesia yield approximately 90,776 and 2,617,208 tweets per day respectively. This stream equals approximately 3.1 GB of data per day, but can surge to much higher volumes. In the aftermath of the Chilean earthquake at the beginning of April, the word "tsunami" rose to global prominence and we found ourselves drawing approximately $20 \mathrm{~GB}$ of tweets per day. Collection has gone through periodic problems due to these surges and keyword changes, but collection has been continuous for much of March and all of April, 2014. The Indonesia stream does not appear in the Padang TWRsms, but is used by the research team for refining the overall TWRsms process.

Collected tweets are formatted as JSON, a lightweight format consisting of lists of key: value pairs, where the contained values may be lists of multiple values or additional key: value pairs. For instance, the key "text" maps to the text of the captured tweet, the key "user" maps to a set of key: value pairs describing the user who posted the tweet. Structural syntax is generally kept to a minimum, consisting of the names of keys and delimiters indicating whether a particular value is a list of values, a list of key: value pairs, or a single value. Even so, approximately $53 \%$ of the tweets that we collect generally consist of structural information. Twitter also provides a number of keys that are not likely to be needed for your analysis in the long term. For example, both hashtags and the particular indices in the text from they were extracted are included. It is likely that for many analyses only the hashtags will be necessary. The user can use the hashtags provided in the JSON, 
validate their presence against the indices provided in the JSON, or write their own code to locate the hashtags. In the end, some of the provided data will be discarded. To handle this excess data, we turn to the next phase of our workflow.

At this point, our experience suggests that the best practice would be to use just a npunding-box to collect tweets - to ensure coverage. And to do a second collection with just user ids - to ensure getting official tweets. However, most of the tweets collected would then have little relevance to disasters. During the planning and early warning phases, this is fine as the point of the collection for those phases is to estimate the population and its location, and to track official warnings. However, during a disaster and in its immediate aftermath, the key will be to limit the tweets to just those of interest. We discuss under management how this can be done. As a final point, we note that the Twitter community is continually evolving; hence any terms being tracked, user IDs, and to a lesser extent the npunding-box will need to be adaptively modified. For instance, if one of the Twitter accounts being followed for Indonesia appears to have stopped publishing tweets, or if is taken over by spammers, it should be removed from the search queries. Correspondingly, if we repeatedly capture tweets from a user who often uses our sampled keywords, we could add the user to the general list of accounts from which we sample because, the comments might be useful, even if one of the established keywords is not used. By adapting our sample, we can better collect and/or ex post facto identify the tweets that we think actually matter.

\section{2 - Management}

In this phase, we focus on cleaning the aggregated data. Summary statistics are stored as part of TWRsms rather than the raw data in order to reduce storage costs, yet retain a meaningful subset of the data. The data is also transformed for ease of use with particular analytic tools such as ORA. Another reason for only storing summary statistics has to do with the ethics of collecting individual data. Although the data collected is public at the time of collection, individuals can decide to make their data private. By storing only summary data, we can retain accurate summaries without keeping specific tweets or individual records; thus effectively removing personally identifiable information issues and complying implicitly with future wishes to have data become private.

It is safest to manipulate the Twitter JSON that you are collecting in a separate location from where you have been archiving it. The best practice is to copy the data to a temporary location for cleaning and then move it to the final storage location. This final storage area could be a database, or it could be a collection of flat files. In either case, performing the cleaning and the transfer will require some sort of middleware.

In our particular case, this middleware consists of a set of Python scripts and a Java management program. When TweetTracker collates tweets, it stores them in a set of flat files in a user-specified folder. We use a Python script to copy these files to a temporary working directory, removing the originals after the transfer. Our script cleans the working data, copying it to a storage location, and then purges the working files. This process reduces the disk required at any time close to that required by the original files.

The cleaning elements of our Python script (and of the process generally) are partly necessitated by the vagaries of the raw data stream, The streaming API often provides a raw data stream that is messy; the JSON collected from the API can be mangled or incomplete; it may be truncated or missing particular keys, and it may include duplicate tweets. The streaming API periodically provides users with the number of tweets that have 
been omitted from the sample, and records of disconnections and reconnections to the stream. These records can provide additional information, but can cause problems for tools that only expect JSON that maps to tweets. If such a tool is being used, this JSON will need to be purged. We also note here that Twitter periodically updates the standard for the JSON that defines a tweet, and if you are using a data warehouse service to collate Twitter JSON the provider may add additional key: value pairs to the data. If the analysis tools are expecting a particular data structure, such changes may cause the tool to discard collated tweets. These additional fields may need to be purged from the data structure. Thus, our Python script purges both malformed tweets and Twitter's periodic warnings about disconnects and counts of missed tweets. This cleaning process also involves purging tweets that are not of interest and reorganizing the tweet JSON by date.

The cleaning elements of our Python script are also partly necessitated by our desire to purge all unwanted tweets from the collection. The streaming API will return tweets that match any parameters specified in the search criteria provided. For early warning and planning we would ideally like all tweets from the region of interest, with the geo-tag for each of these. As Carley et al (this issue) notes, this is not possible. We note, that even the most comprehensive collection will contain tweets not in the region and tweets that are not by humans; e.g., bots. Thus, the first phase of removing unwanted tweets is to simply remove those tweets that are not from the region and/or are by bots. We can safely discard any tweets that have latitudes or longitudes that are outside the region of interest. Twitter users can also set their preferred time zone; since Indonesia is located within the UTC +7 , $\mathrm{UTC}+8$, and $\mathrm{UTC}+9$ time zones -Padang is in $\mathrm{UTC}+7-$. We can generally discard tweets by users who fall outside of this time zone. Cleaning strategies based on latitude and longitude and/or time zone can have markedly different results depending on how the data was collected in the first place; e.g., for the Indonesia data collection using this cleaning strategy $8.72 \%$ the tweets were deemed to be not-relevant, whereas for Padang $38.24 \%$ determined to be not relevant. The reason is that, when a npunding-box plus terms are used in collection a higher fraction of tweets that contain the terms as opposed to being from the region will be selected as the number of tweeters in the region is smaller. Hence, since there are fewer Tweeters in Padang than in Indonesia as a whole, more of the tweets from this collection strategy for Padang will be irrelevant than for Indonesia. Collecting only on the npunding-box will actually reduce the number of irrelevant tweets collected. Using this strategy only about $2 \%$ of the tweets appeared to be irrelevant. As to bots, although we have not yet implemented this in TWRsms we note that bots can account for a large fraction of tweets - sometimes up to $25 \%$ of the tweets. One study estimates that over half of all Twitter accounts are bots. ${ }^{8}$ The tweets by these bots can completely dominate the data and statistically impact what can be learned (Morstatter et al, 2015; Wei, 2015). Collection via a npunding-box may reduce the number of bot generated tweets but it does not eliminate them. Machine learning tools that identify and remove bots maybe the best solution. Such tools are not currently in TWRsms; however, they are planned for the next version.

As our cleaning script moves tweets to the collation area, it regroups them based on their timestamps, with one file per hour. This simplifies the archiving process and helps to keep the data organized. After deleting tweets with bad geotags or inappropriate time zone

\footnotetext{
8 http://blogs.wsj.com/digits/2014/03/21/new-report-spotlights-twitters-retention-problem/
} 
offsets, we have approximately 40,291 tweets per day in our Padang collection, and approximately 1,094,989 tweets per day in our Indonesia collection. (44.39\% and 41.84\% of the original values, respectively.)

Our second middleware program reduces the size of the collected data by converting it from JSON to DyNetML, an XML specification used by the ORA network analysis tool

This reformatting further reduces the memory footprint of the data; one hour's worth of twitter JSON, transformed to DyNetML, is approximately nine percent of its original size; this is true even though the transformation extracts a number of network relationships from the data. (See Appendix B for list of the network features present in a DyNetML file.)

When converting tweets to DyNetML we perform one additional transformation: "unpacking" retweets. In the Twitter JSON format, when a user retweets a tweet that another user has said, the JSON includes a copy of the original, bundled tweet. We extract these bundled tweets and use them to flesh out our collection of data. This is a useful trick for increasing the richness of the data when conducting a longitudinal analysis. As collections continue, one can flesh out the amount of past data that has been retrieved to build more complete pictures of the tweets on any given day of collection.

That said, the earliest tweets extracted using this method can predate the rest of the data by weeks, months, or even years: in our Indonesia collections, we have found retweets of tweets originally sent in 2008! This is, to great extent, an artifact of how Twitter structures its data: if a user is retweeting something that another user has retweeted, the JSON will point back to the original user's tweet, not the tweet being referenced. While this function provides some useful information about the amount of time that tweets can last within the system, it provides an inaccurate picture of the propagation of information across the network of tweeters. If one uses unpacking, it may be simpler to set a threshold date and discard any unpacked tweets that fall before it. The first day of your collection is a natural choice for such a threshold.

The final step of this portion of the analysis is to archive the data through a backup system, or a RAID setup to guarantee redundancy if a hard drive fails. It is useful to keep copies of collected tweets on multiple machines for additional security.

In our case, we keep the machines on which we are storing data on a backup plan provided by the We archive the JSON and the extracted DyNetML using LZMA compression to reduce their memory footprint. The large amount of redundant data in each JSON file makes compression very efficient, reducing the total footprint to between four and five percent of its original size.

Regardless of the nature of the intended analysis, deleting unnecessary features from data is important for managing the data planned for storage. This action will significantly reduce the amount of space needed for data storage. Transforming the Twitter data so that it works with the selected analysis tools generally saves space by removing unnecessary JSON elements when moving it into the tool's preferred format, but this will depend on the format's specification. Compressing and archiving tweets -provided that you delete the uncompressed files- can further reduce the space footprint.

Note, that the only public data available is the set of summary statistics. The underlying tweets, that were cleaned are still available to the research team, but are not sharable legally by the terms of Twitter. We examined whether the set of keywords (Appendix A) could 
be used to remove irrelevant tweets; i.e., all tweets not containing those terms. Our analysis revealed that very few tweets contained those terms so removing those that did not contain them made the twitter data base not useful for estimating where the populations location.

Ideally, at this point, the comprehensive set of tweets would be cross-classified into disaster related areas - e.g., those relating to the need for water, food, shelter, missing individuals, fraud, etc. and statistical summaries for such tweets kept. This would be done using either a lexical approach using terms such as in Appendix A, or better yet, using a machine learning algorithm customized for the community. We also found that many tweets that actually referred to disaster related issues were removed if a straight lexical approach was used, and that many of the tweets containing these terms were not disaster related. We note that such cleaning to identify only disaster related tweets is most useful for providing guidance during a disaster and in its immediate aftermath; it is not useful for planning or for identifying local opinion leaders. While anecdotal evidence suggests that tweets related to disasters are quite infrequent in non-disaster times (possibly less than 1\% of tweets), that the number increases dramatically during a disaster and its aftermath, but that even in those periods disaster related tweets may account for less than $50 \%$ of the tweets in that region. Future research needs to examine this in more detail.

Once extraneous data have been removed and the data archived, it is possible to move on to analysis.

\section{3 - Analysis}

The nature of the analysis will depend on the problems being addressed. For instance, in our implementation we are confronting three distinct challenges with different time frames: planning support, early warning, and research. For planning support TWRsms provides planners with a snapshot of the recent tweetosphere for the area to support. This covers, among over things, where and when tweeting is active thus providing insight into population movement, who are the active and critical tweeters thus providing ideas on who to contact in emergencies, and indicators of concern with disaster related impacts which provides an indication of pre-awareness and post-response to a disaster. For early warning TWRsms provides immediate advice to individuals in danger from a tsunami, after the BMKG early warnings. For researchers, TWRsms provides historical archives of summary information on twitter usage for the region of interest.

Planning: The small window of opportunity for warning citizens of an oncoming Tsunami means that prior information on likely patterns of behavior must be used in planning. We want to be able to provide information on the pattern-of-life in the Padang region. Census based systems provide information on the number of people who live, or work, in a particular section of a city. However, people move through the city during the course of their daily activities. Hence, census based systems do not provide guidelines for what fraction of the population really is where when. This can be quite critical; e.g., a census system might suggest that $50 \%$ of people live in an are above where even the most severe Tsunami would reach; yet, fail to provide the insight that only $5 \%$ of the population is at home during the early morning as they are all of at work, school, or shopping in areas that are at ocean level and likely to be inundated. Twitter has the potential to help us address this gap. Although it is not a full census of the population; it does provide guidance as to which parts of the city are more active when. As such, the data can be used to estimate which sections of the city and what fraction of the population is at risk better than a census only estimate. Key information about centers of population mass at different times of day, 
days of week, and so on support determining where most support needs to be provided. The risk is that there may be very under-represented groups who have patterns of behavior completely distinct from those on Twitter. Another aspect of planning is assessing the extent to which the population is aware of or concerned with standard disaster impacts such as issues of food/hydration, shelter, missing persons, or fatalities. We want to be able to track attention to such issues in the tweets. However, that content analytics is not currently done.

Early Warning: When a potential tsunami is detected, warnings can be broadcast to Indonesian citizens of Padang approximately 20 minutes in advance of the wave's arrival. During this warning phase, we want to be able to provide individuals with useful information being broadcast on Twitter and the names of Twitter users who are providing key information and sending out useful alerts. The risk is that access to the internet may be compromised by the earthquake that has generated the tsunami, and so Twitter may not be functional in large sections of the city. We note that after the tsunami, twitter based tools are likely to only be available once cell towers have been re-stood up and, at least, battery power restored.

Research: In the long term, we want to show trends about the use of twitter in disaster situations both pre-, during and after a disaster and to build models of language that can be used to identify particularly salient tweets and to remove tweets that are redundant. Being able to classify tweets by content would help better meet the needs of disaster response; however, at this point, that is not done. Thus in the short term, as noted under management, we have erred on the side of keeping all tweets in the region of interest for the time period of interest. We also want to use our archive of tweets collected prior to, during and in the aftermath of tsunamis for training exercises; we want to develop a system for "playing back" tweets so that individuals can respond to it. In this case, cross-classifying tweets by the concern evinced with disaster related issues, cries for help, and the emergence of fraud in the aftermath of the disaster are items where long term twitter analytics could help.

The analysis methods for each aspect (planning, warning and research) must be decided as a function of how the tweets will be employed. If working on data for immediate consumption, it may be necessary to cull drastically the information being parsed, perhaps down to just message contents or message contents and usernames, search for meaningful keywords, and then present the content. We are still developing our own tools for immediate analysis. Given the scenario, and the nature of Indonesia and disasters, we are focusing on three classes of metrics. First, general pattern of life statistics indicating who is tweeting from where when. Second, awareness and concern statistics indicating the prevalence of tweets and who is tweeting about keywords and phrases that have been associated with disaster. Third, key actor identification statistics indicating who is influential and capable of disseminating messages at various times of day and regions to varying communities.

If you are planning on looking at data collected over a longer period of time you may want to try and build predictive statistical models, and to extract additional information about users by inspecting their lists of followers or by extracting additional tweets for individuals of interest by searching their accounts. In our case, our long-term analysis methods focus on running two different network analysis reports on our data for various intervals of time; a "report" is the term used by ORA to describe a bundle of several different network metrics. We run these reports on our hourly bundles of files, grouping 
our generated DyNetML files into hourly, daily, weekly, four-week and monthly bundles. All reports are run on an hourly basis; on a given day, we will generate 24 versions of each report. (Excepting the monthly, which is calculated once at the start of the month.) Because we have not yet had to discard any of our uncompressed DyNetML, it is relatively simple for us to re-run reports for any particular time interval.

The first report that we compute is the "Twitter Report". It generates a number of aggregate statistics about tweeters who generated the logged tweets at both the individual level (most tweets logged per users, most retweeted users, etc.) and the network level. (Closeness centrality) Network analysis has been used in prior research on Twitter to try and determine lines of influence, key actors, and points of contention, as well as to characterize the general community of Twitter users (Carley et al, 2014; Hsu \& Park, 2011). While a potent tool for understanding Twitter data, traditional network metrics in the context of Twitter often do not equate to what they previously meant. The ORA Twitter report which is used in TWRsms calculates a number of additional summary statistics around the individual words used in tweets, and the distribution of the locations of the geotagged tweets. (See Appendix C.)

The second report that we compute as part of TWRsms is ORA's Key Entity Report. It generates a number of general network statistics that can be useful within the context of the Meta-Network ontology. By importing the Twitter data into ORA, we generally slot it into this ontology. Because this report focuses on general metrics, it makes some calculations that are redundant with those that have been made in the Twitter Report. (See Appendix D)

Once analysis is complete, it's important to provide a reasonable interface for navigating the data, so that the target audience can examine it, whether they are analysts or the general public.

\section{4 - Presentation}

The intended audience and purpose of the analyses will dictate what results are presented. If one is working in concert with other researchers, it may be simpler to share files of results on a single machine, or store them in a private database. If the general public will interact with the results, they may be presented on a web page that uses D3.js or other tools that will allow manipulation of the data from online (Bostock et al., 2012). If the public only needs to see the results, a static interface takes less effort to create.

Like our analyses, our presentation of results is intended to cater to different sets of needs. We have developed those aspects of presentation that cater to the planning and research, and not those for early warning. All report results described in the analysis section are stored on a web server. A private website allows users to navigate a tree structure containing each report. Users can select particular reports, navigating up and down the tree.

The design of the current data and historical data sections support the presentation of multiple reports. Each of these reports contains summary information for a specific time period based on the tweets collected for that time period. Note, only the most recent data is stored in detail. Historical data is rolled up into summaries, first by day, then by week, then by month. Yearly rollups are being considered. These rollups support research outside the bounds of the needs of tsunamic warnings.

The reports available in TWRsms are generated by the ORA system. To date, there are two major reports being used - the twitter report and the key-entity report. Each of these reports contains detailed assessments that helps the user understand what is being said, who 
is saying it, who is trusted, and so on given the wealth of twitter data available. In Figure 7, a portion of one of these ORA reports is shown. This specific portion related to the "Analysis of Tweeters", in which the significance of different users based on their activity is calculated. In Figure 7, the data shown is from the Padang collection for April 14. The full report can be accessed from the web site.

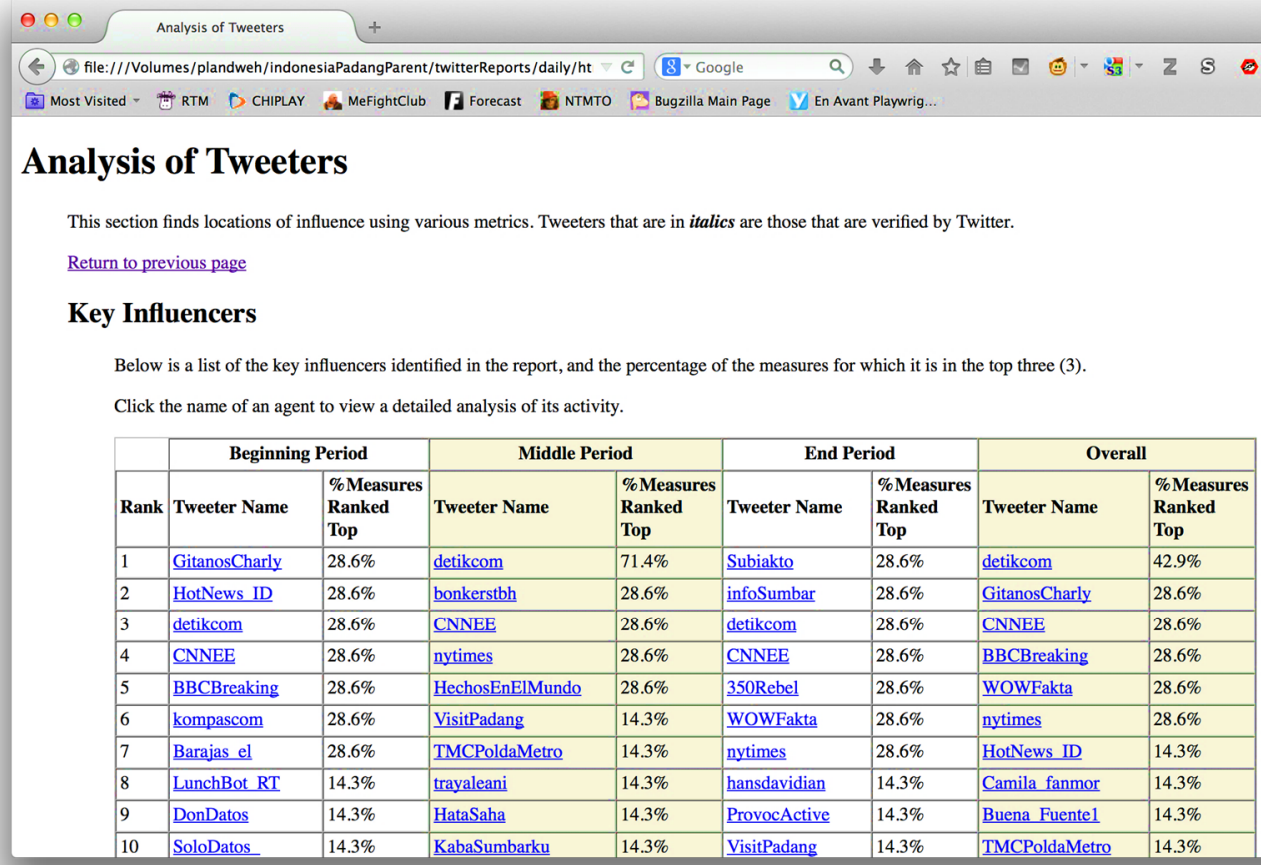

Figure 7. Illustration showing a portion of a sample report from ORA using the Padang collection for April 14.

With respect to early warning, we also want to present any useful information that can be gleaned from Twitter to individuals immediately at risk from a tsunami or enduring in a tsunami's. Our site should serve as a location for individuals to check out what the recommended response is and to identify whom to contact.

Finally, for research, we expect that our site should serve as a useful location for individuals to check for information about the availability of food, the names of missing persons, the conditions of roads, and other information. At its heart, this presentation will include a list of tweets, sorted into different categories of disaster-related tweets, lists of individuals who are providing useful information, and a map of locations from which relevant tweets have been sent or that have been referenced in relevant tweets. To facilitate this, we will be developing a set of metrics for evaluating the relatedness of different tweets to disaster. At present, we have completed research to identify a set of potentially useful disaster terms that can be used as a basis for the analyses that we will eventually present. We note however, that such usage will only occur once power and cell towers are restored. 


\section{5 - Integration}

In general, systems for disaster mediation are not used in isolation. As noted, they need to be used in concert with many other tools. To an extent we have already described points of interoperation with other systems. We highlight here several critical points.

Systems such as TWRsms have to be designed with interoperability in mind. APIs, use of common data formats (if they exist), input-output procedures are needed. That being said, there are additional details that need to be considered: interoperation level, type of interoperation and organizational/legal issues. Each of these is now illustrated.

Interoperation level refers to whether two systems will be strongly or loosely coupled. In TWRsms - the integration with the data provider is a tight coupling e.g. redisplaying tweets, and using a tracker such as TweetTracker to collect tweets. When tightly coupled, changes in the providers API, or rules of use, will impact the stability of new systems such as TWRsms. Further, end maintainers will need to get their own access to such tools. When loosely coupled, data is shared between systems using a common format. TWRsms is loosely coupled with potential research tools as it outputs data in a standard CSV format.

Type of interoperation refers to whether the systems are used as a loose confederation, or whether one becomes a component in the other, or completely integrated in the code of the other. In general, full integration is to be avoided due to cost of development and maintenance, inflexibility, and IP issues. In building TWRsms, ORA, e.g., is treated as a component within TWRsms. Since TWRsms is a web system and ORA a thick client data processing system this required developing a process of data storage, automated batch processing, and support for result uploading. Challenges linking web and non-web system are key to the future of this type of system development.

Organizational and legal issues refers to a panoply of concerns impacting what data can be collected, stored, and shared. As well as concerns over jurisdiction. In the case of systems using Twitter, the policies of Twitter actually preclude sharing of the actual tweets. Thus to move data into the overall sea and land based system that TWRsms is meant to be part of, the data can be shared only in the form of summaries - such as number of tweeters per region. This limits the kinds of questions that can be addressed; e.g. TWRsms cannot support tools that want to know the exact location of a specific individual. As another example, although in principle TWRsms could send out a general tweet that a tsunami was coming, the authority to do so is not officially vested in the this tool; but in BMKG.

\section{5 - Ongoing and Future Social Media Challenges}

The study of social media with respect to early warning and planning for disasters presents several key challenges. The first is that many streams of social media data are only available in a limited form. For example, Facebook provides limited access to data and tightly controls collection of it through its terms of service. While the Twitter Streaming API can be accessed freely, the nature of the sampling makes it unhelpful as a general representation of the complete collection of tweets (Morstatter et al., 2013). The 1\% cap imposed by Twitter on its publically available stream means that potentially valuable tweets go uncollected. If the true volume of tweets using a given keyword exceeds the $1 \%$ threshold, the API will return a nonrandom sample that potentially misrepresents relative frequencies of these terms in the twittersphere. One way to deal with this problem is by adaptively selecting keywords based on new information that arrives, and to continue to try and do better at representing the whole. Another is to concede that a completely 
representative sample cannot be obtained, and so focus on problems where representativeness of the whole isn't critical. In the case of our ongoing work on Indonesian tsunamis, it is important to try and capture as many tweets as possible that contain information related to the tsunami, but knowing what fraction of the twittersphere is made up of tsunami-related tweets is unimportant. For an analysis of the Indonesian Twitter we collected see Carley et al., this issue, and Carley et al., 2015.

Second, as pointed out above, while Indonesians are relatively heavy users of Twitter and represent an impressive one or two percent of the global Twitter stream, this is mostly a consequence of Indonesia's population rather than high average usage. Given that as of 2012 only $15.4 \%$ of Indonesians had Internet access at all, there is a limit to how much of the population will be represented on Twitter. There are also linguistic challenges; most of the infrastructure for analyzing Twitter, including thesauri of keywords and sentiment dictionaries, are designed around English and not Bahasa Indonesian (let alone any of Indonesia's over 700 languages). There is some emergent literature in English by Indonesian researchers on methods of analyzing Indonesian-language tweets that will be accessible to the existing community of Twitter analysis infrastructure development, but it remains a significant challenge (Wijaya et al., 2013; Lunando \& Purwarianti, 2013; Naradhipa \& Purwarianti, 2012)

Third, analyzing the results of Twitter data is difficult and markedly affected by collection methods as is seen in Carley et al, this issue. We currently collect tweets using only a Geobox. We note that less than $10 \%$ of all tweets tend to be geotagged. This fact, in conjunction with the fact that many Indonesians do not have access to the internet means that, TWRsms cannot be used to track the exact movement of the entire population. It can, we have done, provide indications of which parts of the city are more likely to be populated at what time of day for a particular day of the week. Systems like TWRsms need to be built to leverage social media, while not over-claiming its value for disaster response.

Lastly, we do not have any good public policy for regulating access to social media in disaster. Treating a privately-controlled service as part of public infrastructure would generally be considered outside the scope of public policy. However, first responder groups and victims are using social media platforms to communicate during real crises. It is not hard to envision a situation where access to these tools and their data becomes critical to the public good. A disconnect between what the government needs, what companies must provide, how the public expects this relationship to work, and what information the public thinks their data reveals can lead to fiascos such as the recent leaks by Edward Snowden about the NSA. A potentially valuable source of information should not be neglected in designing public policy, especially since social media (to say nothing of other new technologies such as drones) will increasingly play a role in saving lives in disasters. Public policy should speak to this growing role and help society to adjust.

\section{6 - Ongoing and Future Technical Challenges}

There are several principal challenges that we continue to face as we expand on this work: managing the flow of data, carrying out analyses on large data, and -in the long termdeveloping enduring and useful presentations and visual analytics.

First and foremost is managing the flow of data. As described in our methodology, we collect an average of $3.1 \mathrm{~GB}$ of tweets per day but periodically see dramatic spikes in quantity. Storing the results of analysis can also be a significant burden. In our 
implementation, we are trying to generate sixteen reports per hour: two report types, two output formats (CSV and HTML) and four time periods. The reports only take up a fraction of the space needed by the data, but they contribute to the general problem. As described earlier, the two principal ways for addressing this problem are compression and reducing the number of features. Zipping files will dramatically reduce storage space. But doing so will also make the original data more difficult to access and over-time analyses hard to run.

The second challenge is tied to the first: large quantities of data make running analyses very difficult. Betweenness centrality, a staple metric for identifying nodes that are good conduits for information, grows cubically in time as network size increases. In our system, we use 3-way-betweenness, which calculates betweenness as a function of all nodes three hops away. This reduces complexity, but also returns a less accurate result.

Another key challenge is developing reasonable displays for data of this sort remains a difficult challenge. The different reports that we run create various static graphs. These are useful, but also very general The New York Times and other organizations will often use D3.js or other libraries to create lush visualizations of different data sets, but such bespoke visualizations are often very hard to generalize to other situations. Researchers are still searching for the perfect blend of these two elements.

A minor challenge is that to be operationally useful, the system has to work within the dominant languages of the region. Prior work, Carley this issue, shows that there are a number of languages in use in Twitter in Padang. Hence, future plans include - translation of TWRsms into these languages. The first translation will be into Bahasa, activate the disaster alert mode, support for data extraction, and support for identification of tweets calling for aid and classification by category of need (e.g., food, water, or shelter).

The fifth challenge relates to extracting meaningful information for planning and response. A key issue here is identification of the needs of the tweeters as they relate to disaster specific features. To do this, those tweets related to specific disaster related topics such as the need for shelter or presence of fraud, need to be identified, classified into type, and the tweeters located so that support can be provided. We are currently developing a new report on awareness in which the tweets are cross-classified by disaster related topic. In this case activity levels, and key actors would be reported. The heart of this analysis will be a tweet census by topic. At present, we have begun developing a set of general categories adapted from research by Vieweg (2012) that can be used to classify the nature of the disaster-related information contained in a tweet. (See Table 1.) Several of the authors collaboratively labeled 1200 tweets collected during Typhoon Haiyan with these new category labels; a pair of authors labeled each tweet, adding all labels that they believed appropriate. Overall, the authors agreed perfectly on the labeling for 571 tweets, and on at least one label for 213 additional tweets. Using the labels on which the raters agreed as a gold standard, we divided each of the 784 tweets into uni-, bi-, and trigrams, lemmatized words, and deleted noise word, bigrams containing at least one noise word, and trigrams containing at least two noise words. The remaining 14,123 n-grams were kept as a key for assigning labels to as-yet-unclassified tweets. Of these n-grams, only 1,517 were observed more than once in our data.

Table 1 displays information on our ten basic labels, the number of tweets in the 1200 rated that were assigned a label by two raters, the number of n-grams derived from these tweets, and the total number of unique n-grams assigned to each label. 


\begin{tabular}{|l|l|l|l|l|}
\hline \multicolumn{6}{|c|}{ Table 1. Prevalence of Tweets Using Disaster Related Terms } \\
\hline Disaster Term & \multicolumn{1}{|c|}{ Tweets } & N-Grams & $\begin{array}{c}\text { Unique N- } \\
\text { Grams }\end{array}$ & \multicolumn{1}{c|}{ Code } \\
\hline Feeding/Hydration & 12 & 537 & 248 & $\mathrm{Fe}$ \\
\hline Sheltering & 2 & 63 & 44 & $\mathrm{~S}$ \\
\hline Fatality & 72 & 1537 & 909 & $\mathrm{Fa}$ \\
\hline Missing Person & 26 & 782 & 591 & $\mathrm{M}$ \\
\hline Harm & 1 & 110 & 0 & $\mathrm{H}$ \\
\hline $\begin{array}{l}\text { Damage/Repair of } \\
\text { Infrastructure }\end{array}$ & 63 & 1367 & 967 & $\mathrm{D}$ \\
\hline Fraud & 2 & 34 & 16 & $\mathrm{CF}$ \\
\hline Crime other than Fraud & 9 & 219 & 178 & $\mathrm{CO}$ \\
\hline General disaster relief & 148 & 3363 & 2579 & $\mathrm{R}$ \\
\hline Solicitations for aid & 143 & 2467 & 1876 & $\mathrm{~A}$ \\
\hline
\end{tabular}

We have experimented with this labeling scheme, and expect to use it in the next version of TWRsms. In the long term, we see metrics derived from this set of categories being integrated into a new report bundling up different network metrics and tweet features for additional reference by analysts.

The sixth, and final challenge is operational use. Twitter-based systems rely on the existence of cell towers, internet access, and power. In natural disasters, those items may become at best over-loaded, and at worse nonexistent for some period of time. Theses physical realities suggest that the system should be physically place at a location not prone to the local disaster, and that the use of the system will be dependent on remote access.

\section{7 - Conclusion}

In this paper, we have described both the general workflow needed for disaster support social media systems and the specific implementation details of an instance of such a system, the TWRsms. TWRsms collects and analyzes tweets in approximately real time, while monitoring the city of Padang Indonesia in order to support early warning and planning for tsunamis. Using current and historical tweeting activity it provides guidance on where the population is, which is valuable when planning evacuation and response. Using near real time data it provides guidance on who the local opinion leaders are; thus making it possible to direct alerts to these individuals and so working within the community to add an extra layer of early warning. Finally, as it tracks what is tweeted in the region it provides information at, for now, a general level about who is saying what and from which sub-regions within the city. Thus, this information can be used to see where populations are that are unlikely to get a twitter warning. The special Twitter report from ORA, when run by region also provides information on what the key issues as evinced by hashtags are at that time in that region.

As noted, TWRsms is a work in progress. We expect it to also support long-term analysis and review of the different tweets created by Indonesians over a prolonged period, thus support city planning. For this to become a reality, technology for sorting and categorizing tweets by context are needed, as are tools for segmenting the tweets into those from citizens, government or official cites, news agencies and corporations. Preliminary 
analysis suggests that for categorizing tweets by context: 1) separation of tweets based on key words is a first step but insufficient; 2) classification of tweets based on hashtags is a first step but insufficient; 3) topic modeling does not produce clear contexts; and 4) clear classifications will require good statistical modeling of the crisis topics and the use of machine learning techniques for classification. In addition, our analysis suggests that a viable first approach for separating most of the non-citizen and non-bot accounts out is to use compendiums of user ids; e.g., the twitter handles of many corporations are fairly static. However, in the long run, machine learning tools are needed to category the tweeters. Such tools are feasible given the strong differences in the way government and official cites, news agencies and corporations use Twitter as compared to the way the average citizen uses Twitter. Adding such technology to tools such as TWRsms is critical for future systems.

While such advanced technologies are key, they do not address all challenges that must be met. We note that while many systems for aggregating tweets have been developed, a number of challenges still confront them. These challenges are partly related to the nature of social media and partly technical. Fundamentally, social media can be difficult to analyze because it consists of a very self-selected fraction of the population. Sub-samples of that fraction, like the Streaming API, can be even more biased and shouldn't be considered representative of the whole. Many of the tools for working with Twitter data are designed for English, so tweets in Bahasa Indonesian and other tongues can be difficult to parse.

The technical challenges that confronts implementing such systems are largely tied to the quantity of textual data containing latent and manifest content that needs to be processed: how can we store it while balancing our need to look at older data? How can we calculate metrics in real time when they require significant computing power, and more so when the data are large? How can we curtail the collection or presentation of the collected tweets to just those that provide value added information (e.g., information that a street is flooded as opposed to thousands of tweets about the same street being flooded). Our solutions to these problems have been to collect all tweets, categorize them, use network techniques to identify key tweets, and to compress our tweets, to store them in more compact files, and to use simple summary statistics and network statistics as the base for analysis. The cloud has obviously been critical for scaling up systems such as ours, and more analysis of this tension is needed. The final challenge is developing good visuals that trade off ease of generation (our report charts) with useful interactive elements. This is another point of tension, like the data tradeoff, and finding the balance between these two issues remains critical.

Other socio-technical issues have to do with the quality of the data available on Twitter vis-à-vis the society that is producing such data. Thus, one should ask, to what extent do those who tweet reflect the underlying population? For Padang and Indonesia, this was addressed in Carley et al, (this issue). When is the information from twitter accurate, upto-date, and useful? This is in part a function of who is using Twitter and the presence of bots. When is the data from twitter redundant with data that can be gotten from other media? This is in part a function of what other media are available and to what extent is Twitter being used to simply talk about or point to the information in this other media. And so on. While we have not addressed these issues directly, we note that systems like TWRsms should be designed with such factors in mind. For TWRsms this has meant 
collecting tweets in a way that affords greater coverage of the population. It means that in future versions automated bot detectors will be used to remove information not from the community. And it means that local official coordinators such as BMKG and major news services are followed.

Still other issues are policy oriented in nature. It is important to note that the Twitter data we use is in the public domain and under the creative commons license. Hence it is possible to ethically and legally identify individuals and message them using mentions. However, systems such as TWRsms must be careful to only mention, and so communicate with, those users whose current profiles are public. If individuals change their profile to private then they cannot be followed and it is not clear whether mentions of them will reach them. We proposed using Twitter to identify those who has disproportionate influence in Twitter in the local community at that time and to contact them so that should they choose to do so, they could help warn the community that a Tsunami was coming. In this sense, Twitter is being used to create a personalized alarm. We also note, that while it would be ethical given the creative commons license to contact these individuals, it might not be reasonable to have any expectation that they will have to pass on the messages. We note that laws on the use of Twitter data are only just now coming into effect, and that there will be cultural norms governing these laws. Future systems will need to be designed to consider the specific legislation and cultural norms for the community that they are trying to assist.

In a very real sense, our understanding of how to leverage social media for effective disaster management is still in its infancy. As noted, there are a number of technical, social and policy challenges. We look forward to the future work that will address these challenges, and developing our own solutions to them as we generate new versions of the TWRsms.

\section{Acknowledgement}

This work was supported in part by the National Science Foundation 00361150115291 with support for Twitter analysis of Indonesia and the Office of Naval Research N00014140737 with support for

The views and conclusions contained in this document are those of the authors and should not be interpreted as representing the official policies, either expressed or implied, of the National Science Foundation, the Office of Naval Research or the U.S. Government.

\section{References}

Ai, F., Comfort L.K., Dong Y. and T. Znati (forthcoming, this issue) A dynamic decision support system based on geographical information and mobile social networks: A model for tsunami risk mitigation in Padang, Indonesia

@ TwitterMedia, “NPR's Andy Carvin Uses Twitter to Debunk A Hoax," \#OnlyOnTwitter. aa1ronham@gmail.com and J. Rosslein, tweepy.

Acar, A. and Y. Muraki, “Twitter for crisis communication: lessons learned from Japan's tsunami disaster," Int. J. Web Based Communities, vol. 7, no. 3, pp. 392-402, Jul. 2011. Anggunia, S.D. and L. Kumaralaita, "How Indonesians Use ICT and Social Media for Disaster Management," discover.isif.asia, 13-Mar-2014. 
Armstrong, C. L. and F. Gao, "Now Tweet This : How News Organizations Use Twitter," Electron. News, vol. 4, no. 4, pp. 218-235, 2010.

Asur, S. and B. A. Huberman, 2010, "Predicting the Future with Social Media," presented at the Web Intelligence and Intelligent Agent Technology (WI-IAT), 2010 IEEE/WIC/ACM International Conference on, 2010, vol. 1, pp. 492-499.

Basher, R., 2006. Global early warning systems for natural hazards: systematic and peoplecentered. Philosophical Transactions of the Royal Society of London A: Mathematical, Physical and Engineering Sciences, 364(1845), 2167-2182.

Birowo, M.O., "The use of community radio in managing natural disaster in Indonesia," Bull. Am. Soc. Inf. Sci. Technol., vol. 36, no. 5, pp. 18-21, 2010.

Bostock, M., V. Ogievetsky, and J. Heer, "D3 Data-Driven Documents," Vis. Comput. Graph. IEEE Trans., vol. 17, no. 12, pp. 2301-2309, Dec. 2011.

Bruns, A. and Y. Liang, "Tools and methods for capturing Twitter data during natural disasters," First Monday, vol. 17, no. 4, Apr. 2012.

Carley, K.M. and J. Reminga, "ORA: Organizational Risk Analyzer," Carnegie-Mellon University, School for Computer Science, Institute for Software Research, Pittsburgh, Pennsylvania, Technical CMU-ISRI-05-109, 2004.

Carley, K.M., J. Reminga, J. Storrick, and D. Columbus, "ORA User's Guide 2013," Carnegie Mellon University, School of Computer Science, Institute for Software Research, Pittsburgh, Pennsylvania, CMU-ISR-13-108, 2013.

Carley, K.M., 2014, ORA: A Toolkit for Dynamic Network Analysis and Visualization, In Reda Alhajj and Jon Rokne (Eds.) Encyclopedia of Social Network Analysis and Mining, Springer.

Carley, K.M., J. Pfeffer, F. Morstatter, and H. Liu. 2014. Embassies Burning: Toward a Near Real Time Assessment of Social Media Using Geo-Temporal Dynamic Network Analytics, Social Network Analysis and Mining, 4:195. DOI: 10.1007/s13278-0140195-3

Carley, K.M., M. Malik, P. Landwehr, J. Pfeffer and M. Kowalchuk, (forthcoming, this issue) Crowd Sourcing Disaster Management: The Complex Nature of Twitter Usage in Indonesia

Carley, K.M., M. Malik, M. Kowalchuk, J. Pfeffer and P. Landwehr, 2015, "Twitter Usage in Indonesia," Carnegie-Mellon University, School for Computer Science, Institute for Software Research, Pittsburgh, Pennsylvania, Technical CMU-ISR-15-109, 2015.

Center For Excellence in Disaster Management \& Humanitarian Assistance, "Indonesia," Center For Excellence in Disaster Management \& Humanitarian Assistance, Hawaii, USA, May 2011.

Chatfield, A. and U. Brajawidagda, "Twitter tsunami early warning network: a social network analysis of Twitter information flows," in Proceedings of the 23rd Australasian Conference on Information Systems 2012, Australia, 2012.

CNN Library, "Tsunami of 2004 Fast Facts," CNN, 23-Aug-2013. .

Coppola, D. J., 2015, Introduction to International Disaster Management, 3nd ed, Butterworth-Heinemann.

Dynes, R.R., Organized Behavior in Disaster. Heath, 1970.

E. Michaels-Ober, twitter. 
Earle, P., M. Guy, R. Buckmaster, C. Ostrum, S. Horvath, and A. Vaughan, "OMG Earthquake! Can Twitter Improve Earthquake Response?," Seism. Res. Lett., vol. 81, no. 2, pp. 246-251, 2010.

Earthquake Engineering Research Institute, "The Magnitude 7.6 Western Sumatra Earthquake of September 30, 2009," Earthquake Engineering Research Institute, Dec. 2009.

Fitzgerald, B., "Most Popular Sites 2012: Alexa Ranks The 500 Most-Visited Websites," The Huffington Post, 09-Aug-2012. .

Gao, H., Barbier, G., \& Goolsby, R., 2011. Harnessing the crowdsourcing power of social media for disaster relief. IEEE Intelligent Systems, (3), 10-14.

Hsu, C. and Han Woo Park, "Sociology of Hyperlink Networks of Web 1.0, Web 2.0, and Twitter: A Case Study of South Korea," Soc. Sci. Comput. Rev., vol. 29, no. 3, pp. 354-368, Aug. 2011.

Kumar, A. and F. Morstatter, "TweetTracker," 2011. [Online]. Available: http://tweettracker.fulton.asu.edu.

Kumar, S., G. Barbier, M. A. Abbasi, and H. Liu, 2011. "TweetTracker: An Analysis Tool for Humanitarian and Disaster Relief," in Proceedings of the 2011 International AAAI Conference on Weblogs and Social Media, Barcelona, Spain, 2011, pp. 661-662.

Libby, Mathew, 2013, How twitter covered the Queensland floods. ABC News, Australia, 4-26-2013, http://ab.co/lf4UCQL.

Lunando, E. and A. Purwarianti, "Indonesian social media sentiment analysis with sarcasm detection," in Proceedings of the 2013 International Conference on Advanced Computer Science and Information Systems, Bali, Indonesia, 2013, pp. 195-198.

Mendoza, M., B. Poblete, and C. Castillo, "Twitter under crisis: can we trust what we RT?," in Proceedings of the First Workshop on Social Media Analytics (SOMA), Washington D.C., District of Columbia, 2010, pp. 71-79.

Morstatter, F., J. Pfeffer, H. Liu, and K. M. Carley, "Is the Sample Good Enough? Comparing Data from Twitter's Streaming API with Twitter's Firehose," in Proceedings of the 2013 International AAAI Conference on Weblogs and Social Media, Boston, Massachusetts, USA, 2013.

Morstatter, F., K. M. Carley, \& H. Liu, Bot Detection in Social Media: Networks, Behavior, and Evaluation. The 2015 IEEE/ACM International Conference on Advances in Social Networks Analysis and Mining (ASONAM 2015). Paris, France, August 25-28, 2015

Munro, R., "Crowdsourcing and the crisis-affected community: Lessons learned and looking forward from Mission 4636," Inf. Retr., vol. 16, no. 2, pp. 210-266, Apr. 2013.

Naradhipa, A.R., and A. Purwarianti, "Sentiment classification for Indonesian message in social media," in Proceedings of the 2012 International Conference on Cloud Computing and Social Networking, Bandung, West Java, Indonesia, 2012, pp. 1-5.

O’ Brien, J.I., yourTwapperKeeper. 540.co, 2013.

Panagiotopoulos, P., A. Ziaee Bigdeli, and S. Sams, “"5 Days in August' - How London Local Authorities Used Twitter during the 2011 Riots," in Electronic Government, vol. 7443, H. Scholl, M. Janssen, M. Wimmer, C. Moe, and L. Flak, Eds. Springer Berlin Heidelberg, 2012, pp. 102-113. 
Sadilek, A., H. A. Kautz, and V. Silenzio, "Modeling Spread of Disease from Social Interactions.," in Proceedings of the 2012 International AAAI Conference on Weblogs and Social Media, 2012.

Sakaki, T., M. Okazaki, and Y. Matsuo, "Earthquake shakes Twitter users: real-time event detection by social sensors," in Proceedings of the 19th International Conference on World Wide Web, Raleigh, North Carolina, USA, 2010, pp. 851-860.

Santos, R.M., D. Mosse, T. Znati, and L.K. Comfort, (forthcoming, this issue) Design and Implementation of a Witness Unit for opportunistic routing in Tsunami Alert Scenarios

Semiocast, "Twitter reaches half a billion accounts; More than 140 millions in the U.S.," Semiocast Publications, 30-Jul-2012.

St. Denis, L. A., A. L. Hughes, and L. Palen, "Trial by Fire: The Deployment of Trusted Digital Volunteers in the 2011 Shadow Lake Fire," in Proceedings of the 9th International Conference on Information Systems for Crisis Response and Management, Vancouver, British Columbia, Canada, 2012.

Sutton, J., "Twittering Tennessee: Distributed Networks and Collaboration Following a Technological Disaster," in Proceedings of the 7th International Conference on Information Systems for Crisis Response and Management, Seattle, Washington, USA, 2010.

swatkat...@gmail.com,_johnaboi...@gmail.com,_mikeispr...@gmail.com, antir..@gmail.com, and adam.bev...@gmail.com, twitcurl.

Twitter, "The Streaming APIs," Twitter Developers, 24-Sep-2012. .

Twitter, Hosebird Client. Twitter, 2013.

United Nations Economic and Social Council, 2012, "The Naming Procedures of Indonesia's Islands", Tenth United Nations Conference on the Standardization of Geographical Names, New York, 31 July - 9 August 2012.

United States Geological Survey, "Largest Earthquakes in the World Since 1900," 11-Apr2012.

Vieweg, S.E. "Situational Awareness in Mass Emergency: A Behavioral and Linguistic Analysis of Microblogged Communications," University of Colorado at Boulder, Boulder, Colorado, USA, 2012.

Waltham, T., "The Asian Tsunami Disaster," Geology Today, 21(1), 2005, pp. 22-26.

Wei, W., K. Joseph, H. Liu \& K. M. Carley, 2015, The Fragility of Twitter Social Networks Against Suspended Users, Proceedings of The International Conference on Advances in Social Network Analysis and Mining

Wijaya, V., A. Erwin, M. Galinium, and W. Muliady, "Automatic mood classification of Indonesian tweets using linguistic approach," in Proceedings of the 2013 International Conference on Information Technology and Electrical Engineering, Yogyakarta, Indonesia, 2013, pp. 41-46.

Xerandy, et al. (forthcoming, this issue) A cost effective, environmentally-aware undersea infrastructure to enhance community resilience to tsunami.

\section{Appendix A: Data Selection Parameters for Padang}

\section{Keywords:}

\section{Locations:}

padang, \#padang

bungus teluk kabung, \#bungustelukkabung 
koto tangah, \#kototangah

kuranji, \#kuranji

lubuk begalung, \#lubukbegalung

lubuk kilangan, \#lubukkilangan

nanggalo, \#nanggalo

padang barat, \#padangbarat

padang selatan, \#padangselatan

padang timur, \#padangtimur

padang utarah, \#padang utarah

pauh, \#pauh

minangkabau, \#minangkabau

andalas university, \#andalas

fauzi bahar, \#fauzibahar

emmahaven, \#emmahaven

teluk bayur, \#telukbayur

bayur bay, \#bayurbay

\section{Politics:}

yudhoyono, \#yudhoyono

boediono, \#boedino

bakrie, \#bakri

sukarnoputri, \#sukarnoputri

iskandar, \#iskandar

matta, \#matta

rajasa, \#rajasa

wiranto, \#wiranto

suhardi, \#suhardi

demokrat, \#demokrat

golkar, \#golkar

pdip, \#pdip

pdi-p, \#pdi-p

pkb, \#pkb

pks, \#pks

hanura, \#hanura

gerindra, \#gerindra

pancasila, \#pancasila

\section{Related to Disaster:}

tsunami

makanan

bekal

lauk

makan

air

minuman

halte 


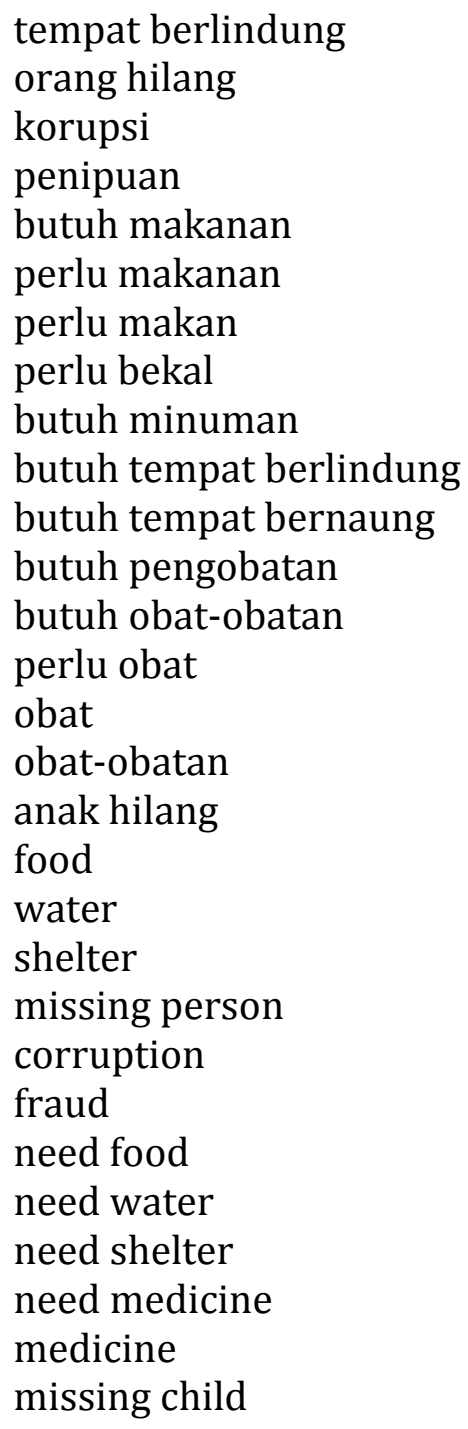

\section{Bounding Geobox:}

Southeast Corner: $(-1.05,100.25)$

Northwest Corner: $(-0.75,100.5)$

\section{Appendix B: The Properties of DyNetML imported from Twitter}

Original tweets are extracted from retweets.

Tweets are aggregated into Meta-Networks by hour

Each Meta-Network consists of the following node sets and networks:

Node sets:

- Agents (Tweeters)

- Events (Tweets)

- Knowledge (Concepts)

\section{Networks:}

- Agent x Agent - Mentions

- Agent x Agent - Retweeted By

- Agent x Event - Sender

- Event x Agent - Mentions

- Event x Knowledge

- Event x Event - Retweeted By 


\section{Appendix C: ORA Twitter Report Metrics}

Tweeters:

- Follower count

- Whether or not the account has been verified by Twitter.

- Times that this user retweet others

- Other tweeters reached by this user, either directly or through retweets.

- Times mentioned by others

- Times retweeted by others

- K-closeness to other users on the union of the networks of tweeters who have been retweeted and tweeters who have been mentioned, for $\mathrm{k}=3$.

- K-betweenness to other users on the union of the networks of tweeters who have been retweeted and tweeters who have been mentioned, for $\mathrm{k}=3$.

- Density of the retweet network over time

- Density of the mention network over time

- Density of the tweeter $x$ concept network over time

\section{Locations:}

- Tweets per longitude/latitude pair

\section{Tweets:}

- Retweet count

- Total retweets logged over time

- Total tweets logged over time

- Total mentions in tweets over time

- Total unique concepts in tweets over time

- Density of the network

\section{Appendix D: ORA's Key Entity Report Metrics}

\section{Agents (Tweeters):}

- Counts of mentions by other tweeters

- Counts of retweets by other tweeters

- Counts of tweets with 'exclusive' and 'relatively exclusive' ties to Tweets

- Total users retweeted by other users over time

- Total users mentioned by other users over time

\section{Knowledge (Concepts):}

- Total times each concept is used

\section{Events (Tweets):}

- Total times each tweet is retweeted

- Total tweeters mentioned in each tweet

- Total concepts used in each tweet

- Tweeters who are mentioned in the most tweets

- Concepts that are used in the most tweets 
- Tweets that are most often retweeted

\section{Locations:}

- Locations from which the most tweets were sent 


\section{Tsunami Warning and Response Social}

\section{Media System}

\section{twitter}
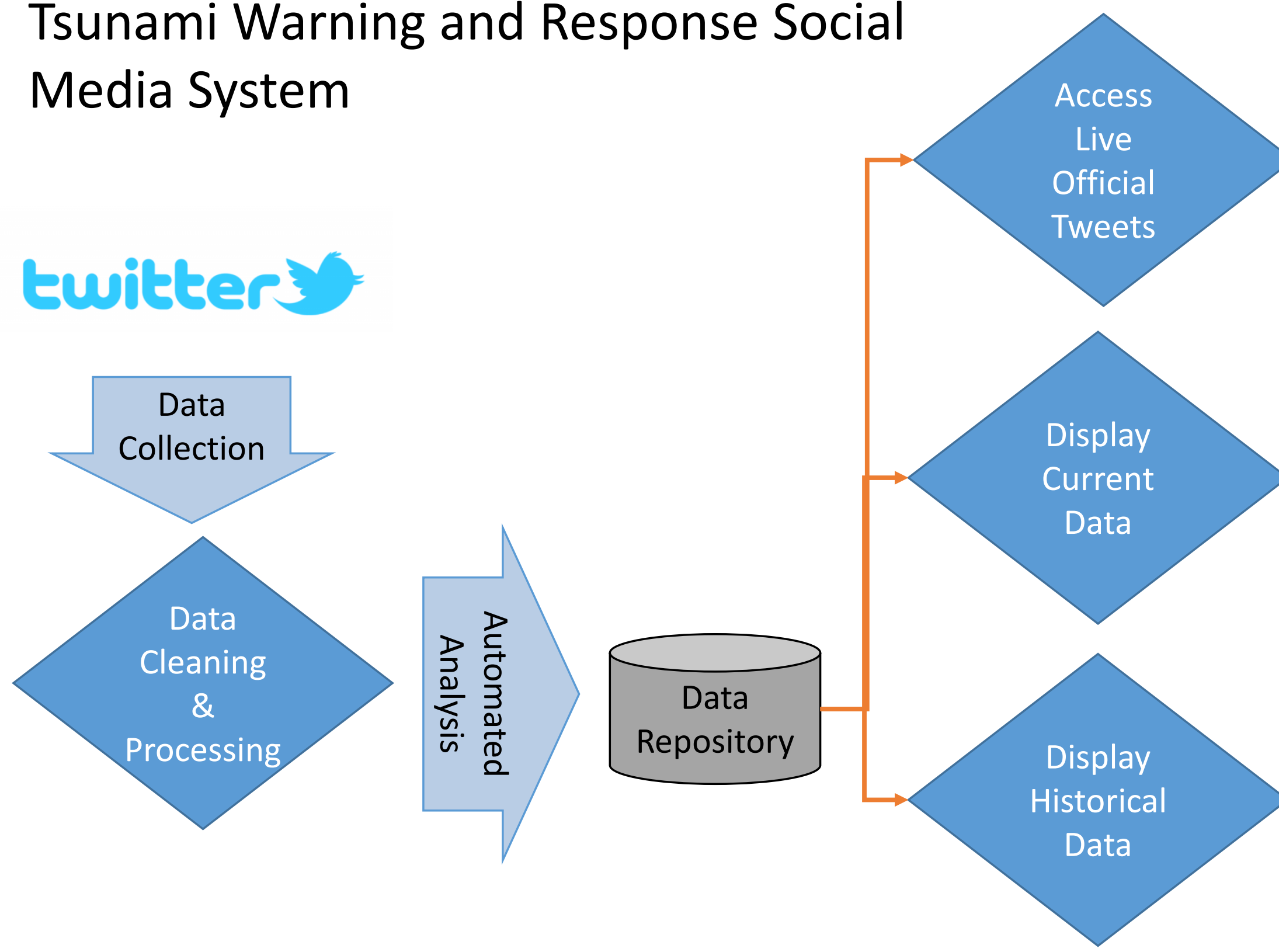

\section{BMKG Tweets}

Social Network Analysis Identifying Key Actors

Pattern of Life Analysis Expectations for Number Impacted 